\title{
Existence and global exponential stability of an almost periodic solution for a host-macroparasite equation on time scales
}

\section{Zhijian Yao*}

\section{"Correspondence:}

zhijianyao@126.com

Department of Mathematics and

Physics, Anhui Jianzhu University,

Hefei, 230601, China

\section{Springer}

\begin{abstract}
This paper deals with almost periodicity of a host-macroparasite model on time scales. By applying the contraction mapping fixed point theorem and exponential dichotomy, we establish sufficient conditions for the existence of a unique almost periodic positive solution. Moreover, we investigate global exponential stability of the almost periodic positive solution by means of the Gronwall inequality.

MSC: $34 \mathrm{~K} 14 ; 34 \mathrm{~K} 20$

Keywords: host-macroparasite model on time scales; almost periodic solution; exponential dichotomy; contraction mapping; global exponential stability
\end{abstract}

\section{Introduction}

The dynamical behavior of biological systems has become a very important and hot research topic. As we know, many human diseases, particularly in tropical and subtropical regions, arise from infection by macroparasites or metazoan organisms. These organisms tend to have much larger generation times and more complex life cycles than microparasites. In life cycles there are two or more obligatory host species together with the final host (humans). Macroparasitic infections are generally chronic in form and they are more a cause of morbidity than mortality and tend to be persistent in character in areas where they are endemic. The final hosts of parasites are usually humans (the hosts in which the parasite attains reproductive maturity) and they gain entry to the definitive host as a consequence of developmental changes which normally occur before the organism arrives at its preferred site and attains reproductive maturity. In [1], Kostitzin constructed a model of the flow of hosts among a series of classes denoting different infection states defined by the number of parasites harbored. For further research in this direction, see Hairston [2], Tallis and Leyton [3], and Leyton [4].

In the study of transmission dynamics of the macroparasite model, there exist two variables $M(t)$ and $L(t)$, where $M(t)$ is the number of sexually mature worms in the human community and $L(t)$ is the number of infective larvae in the habitat. In [5], May and Anderson focused on the dynamics of the adult worms $M(t)$, they proposed the hostmacroparasite model

$$
M^{\prime}(t)=-\beta M(t)+\frac{\alpha M(t)}{[1+\delta M(t-\tau)]^{N+1}} .
$$

(0) 2015 Yao; licensee Springer. This is an Open Access article distributed under the terms of the Creative Commons Attribution License (http://creativecommons.org/licenses/by/4.0), which permits unrestricted use, distribution, and reproduction in any medium, provided the original work is properly credited. 
For more details on the derivation of model (1.1), we refer the reader to [5] by May and Anderson. In [6], Elabbasy et al. studied the oscillation of solutions of model (1.1).

As we know, in the real world, some processes vary continuously while others vary discretely. These processes can be modeled by differential equations and difference equations, respectively. However, there are also many processes that vary both continuously and discretely. Thus an interesting and challenging problem arises: How can we model these mixed processes? The theory of time scale calculus and dynamic equations on time scales provides us with a powerful tool for attacking such mixed processes. For example, time scales are believed to provide a good way to understand and control the West Nile virus since time scale calculus and dynamic equations on time scales can bridge the divide between discrete and continuous aspects of the West Nile virus [7]. The calculus on time scales (see [8,9] and the references cited therein) was initiated by Hilger [10] in order to unify continuous and discrete analysis, and it has a tremendous potential for applications and has recently received great attention. The two main features of the calculus on time scales are unification and extension.

The existence and stability of periodic solutions or almost periodic solutions for differential equations and difference equations are very basic and important problems. It is natural to ask whether we can explore such existence and stability problems in a unified way and offer more general conclusions. The study of dynamic equations on time scales can unify and extend the fields of differential and difference equations.

Motivated by the above facts, in this paper, we investigate the following nonautonomous almost periodic host-macroparasite dynamic equation on time scales:

$$
x^{\Delta}(t)=-a(t) x(t)+\sum_{i=1}^{m} \frac{b_{i}(t) x\left(t-\tau_{i}(t)\right)}{\left[1+x\left(t-\tau_{i}(t)\right)\right]^{N_{i}+1}} .
$$

Almost periodicity is more practical and closer to the reality in biological systems [11, 12]. However, to our knowledge, no papers deal with the existence and global exponential stability of a unique almost periodic positive solution for the above model (1.2) on time scales.

In this paper, we aim to establish sufficient conditions that guarantee the existence of a unique almost periodic positive solution of model (1.2) on time scales by applying the contraction mapping fixed point theorem and exponential dichotomy. We also investigate the global exponential stability of the almost periodic positive solution by means of the Gronwall inequality. The results of this paper are new and more valuable in applications, which complement and extend the previously obtained results in $[5,6]$. Our study reveals that, for the existence and stability of almost periodic solutions of differential equations and difference equations, it is unnecessary to prove results for differential equations and separately again for difference equations. We can unify such existence and stability problems in the frame of dynamic equations on time scales.

\section{Preliminaries}

In this section, we present some basic definitions and preliminary results from the calculus on time scales and almost periodic functions. For more details, see $[8,9,13,14]$. 
The symbol $\mathbb{T}$ denotes a time scale, which is a nonempty closed subset of $\mathbb{R}$. Some examples of such time scales are

$$
\mathbb{R}, \quad \mathbb{Z}, \quad \bigcup_{k \in \mathbb{Z}}[2 k, 2 k+1], \quad \bigcup_{k \in \mathbb{Z}} \bigcup_{n \in \mathbb{N}}\left\{k+\frac{1}{n}\right\} .
$$

Definition 1 The forward and backward jump operators $\sigma, \rho: \mathbb{T} \rightarrow \mathbb{T}$ and the graininess $\mu: \mathbb{T} \rightarrow \mathbb{R}^{+}$are defined, respectively, by

$$
\sigma(t)=\inf \{s \in \mathbb{T}: s>t\}, \quad \rho(t)=\sup \{s \in \mathbb{T}: s<t\}, \quad \mu(t)=\sigma(t)-t .
$$

A point $t \in \mathbb{T}$ is called left-dense if $t>\inf \mathbb{T}$ and $\rho(t)=t$, left-scattered if $\rho(t)<t$, rightdense if $t<\sup \mathbb{T}$ and $\sigma(t)=t$, and right-scattered if $\sigma(t)>t$.

If $\mathbb{T}$ has a left-scattered maximum $m$, define $\mathbb{T}^{k}=\mathbb{T}-\{m\}$; otherwise, set $\mathbb{T}^{k}=\mathbb{T}$.

If $\mathbb{T}$ has a right-scattered minimum $m$, define $\mathbb{T}_{k}=\mathbb{T}-\{m\}$; otherwise, set $\mathbb{T}_{k}=\mathbb{T}$.

Definition 2 A function $f: \mathbb{T} \rightarrow \mathbb{R}$ is right-dense continuous provided it is continuous at right-dense points in $\mathbb{T}$ and its left-side limits exist (finite) at left-dense points in $\mathbb{T}$. If $f$ is continuous at each right-dense point and each left-dense point, then $f$ is said to be a continuous function on $\mathbb{T}$.

Definition 3 For $f: \mathbb{T} \rightarrow \mathbb{R}$, we define $f^{\Delta}(t)$ to be the number (if it exists) with the property that for any given $\varepsilon>0$, there exists a neighborhood $U$ of $t$ such that

$$
\left|(f(\sigma(t))-f(s))-f^{\Delta}(t)(\sigma(t)-s)\right|<\varepsilon|\sigma(t)-s| \quad \text { for all } s \in U .
$$

We call $f^{\Delta}(t)$ the delta (or Hilger) derivative of $f$ at $t$.

If $F^{\Delta}(t)=f(t)$, then we define the delta integral by

$$
\int_{r}^{t} f(s) \Delta s=F(t)-F(r) \quad \text { for } t, r \in \mathbb{T} .
$$

Definition 4 A function $p: \mathbb{T} \rightarrow \mathbb{R}$ is called regressive provided $1+\mu(t) p(t) \neq 0$ for all $t \in \mathbb{T}$.

The set of all regressive and rd-continuous functions $p: \mathbb{T} \rightarrow \mathbb{R}$ will be denoted by $\Re=$ $\Re(\mathbb{T}, \mathbb{R})$.

We define the set $\Re^{+}=\Re^{+}(\mathbb{T}, \mathbb{R})=\{p \in \Re: 1+\mu(t) p(t)>0, \forall t \in \mathbb{T}\}$.

Definition 5 If $p$ is a regressive function, then the generalized exponential function $e_{p}$ is defined as the unique solution of the initial value problem $y^{\Delta}=p(t) y, y(s)=1$, where $s \in \mathbb{T}$. An explicit formula for $e_{p}(t, s)$ is given by

$$
e_{p}(t, s)=\exp \left\{\int_{s}^{t} \xi_{\mu(\tau)}(p(\tau)) \Delta \tau\right\} \quad \text { for all } s, t \in \mathbb{T} \text {, }
$$

where

$$
\xi_{h}(z)= \begin{cases}\frac{\log (1+h z)}{h}, & \text { if } h \neq 0, \\ z, & \text { if } h=0 .\end{cases}
$$


Definition 6 Let $p, q: \mathbb{T} \rightarrow \mathbb{R}$ be two regressive functions, define

$$
p \oplus q=p+q+\mu p q, \quad \ominus p=-\frac{p}{1+\mu p}, \quad p \ominus q=p \oplus(\ominus q)
$$

Lemma 1 Assume that $p, q: \mathbb{T} \rightarrow \mathbb{R}$ are two regressive functions, then

(i) $e_{0}(t, s) \equiv 1, e_{p}(t, t) \equiv 1$;

(ii) $e_{p}(\sigma(t), s)=(1+\mu(t) p(t)) e_{p}(t, s)$;

(iii) $\frac{1}{e_{p}(t, s)}=e_{\ominus p}(t, s), e_{p}(t, s)=\frac{1}{e_{p}(s, t)}=e_{\ominus p}(s, t)$;

(iv) $e_{p}(t, s) e_{p}(s, r)=e_{p}(t, r), e_{p}(t, s) e_{q}(t, s)=e_{p \oplus q}(t, s)$;

(v) $\left(e_{p}(t, s)\right)^{\Delta}=p e_{p}(t, s)$;

(vi) If $a, b, c \in \mathbb{T}$, then $\int_{a}^{b} p(s) e_{p}(c, \sigma(s)) \Delta s=e_{p}(c, a)-e_{p}(c, b)$.

Definition 7 [13] Let $\Gamma$ be a collection of sets which is constructed by subsets of $\mathbb{R}$. A time scale $\mathbb{T}$ is called an almost periodic time scale with respect to $\Gamma$ if

$$
\Gamma^{*}=\left\{ \pm \tau \in \bigcap_{\Lambda \in \Gamma} \Lambda: t \pm \tau \in \mathbb{T}, \forall t \in \mathbb{T}\right\} \neq \emptyset
$$

and $\Gamma^{*}$ is called the smallest almost periodic set of $\mathbb{T}$.

Definition 8 [13] Let $\mathbb{T}$ be an almost periodic time scale with respect to $\Gamma$. A function $f(t) \in C\left(\mathbb{T}, \mathbb{R}^{n}\right)$ is called almost periodic if for any given $\varepsilon>0$, the set $E(f, \varepsilon)=\left\{\tau \in \Gamma^{*}\right.$ : $|f(t+\tau)-f(t)|<\varepsilon, \forall t \in \mathbb{T}\}$ is relatively dense in $\mathbb{T}$; that is, for any given $\varepsilon>0$, there exists a real number $l=l(\varepsilon)>0$ such that each interval of length $l$ contains at least one $\tau=\tau(\varepsilon) \in$ $E(f, \varepsilon)$ satisfying $|f(t+\tau)-f(t)|<\varepsilon, \forall t \in \mathbb{T}$.

The set $E(f, \varepsilon)$ is called $\varepsilon$-translation set of $f(t), \tau$ is called $\varepsilon$-translation number of $f(t)$, and $l(\varepsilon)$ is said to contain interval length of $E(f, \varepsilon)$.

Remark If $\Gamma=\{\mathbb{R}\}$ and $\mathbb{T}=\mathbb{R}$, then $\Gamma^{*}=\mathbb{R}$, in this case, Definition 8 is equivalent to the definition of almost periodic function in [11]. If $\Gamma=\{\mathbb{Z}\}$ and $\mathbb{T}=\mathbb{Z}$, then $\Gamma^{*}=\mathbb{Z}$, in this case, Definition 8 is equivalent to the definition of almost periodic sequence in [15].

Definition $9[13,16]$ Let $Q(t)$ be an $n \times n$ rd-continuous matrix function on $\mathbb{T}$.

The linear system

$$
x^{\Delta}(t)=Q(t) x(t), \quad t \in \mathbb{T}
$$

is said to admit an exponential dichotomy on $\mathbb{T}$ if there exist positive constants $k, \alpha$, projection $P$ and the fundamental solution matrix $X(t)$ of (2.1) satisfying

$$
\begin{aligned}
& \left\|X(t) P X^{-1}(\sigma(s))\right\| \leq k e_{\ominus \alpha}(t, \sigma(s)) \quad \text { for } t \geq \sigma(s), s, t \in \mathbb{T}, \\
& \left\|X(t)(I-P) X^{-1}(\sigma(s))\right\| \leq k e_{\ominus \alpha}(\sigma(s), t) \quad \text { for } t \leq \sigma(s), s, t \in \mathbb{T} .
\end{aligned}
$$

Consider the almost periodic system

$$
x^{\Delta}(t)=Q(t) x(t)+g(t), \quad t \in \mathbb{T},
$$


where $Q(t)$ is an almost periodic matrix function, $g(t)$ is an almost periodic vector function.

Lemma 2 [13, 14] If the linear system (2.1) admits an exponential dichotomy, then the almost periodic system (2.2) has a unique almost periodic solution $x(t)$ as follows:

$$
x(t)=\int_{-\infty}^{t} X(t) P X^{-1}(\sigma(s)) g(s) \Delta s-\int_{t}^{+\infty} X(t)(I-P) X^{-1}(\sigma(s)) g(s) \Delta s .
$$

Lemma 3 [8] Let $Q(t)$ be a regressive $n \times n$ matrix-valued function on $\mathbb{T}$. Let $t_{0} \in \mathbb{T}$ and $x_{0} \in \mathbb{R}^{n}$, then the initial value problem

$$
x^{\Delta}(t)=Q(t) x(t), \quad x\left(t_{0}\right)=x_{0}
$$

has a unique solution $x(t)$ as follows:

$$
x(t)=e_{Q}\left(t, t_{0}\right) x_{0} .
$$

Lemma 4 [13] Let $c_{i}(t)$ be an almost periodic function on $\mathbb{T}$, where $c_{i}(t)>0,-c_{i}(t) \in \mathfrak{R}^{+}$, $\forall t \in \mathbb{T}$ and

$$
\min _{1 \leq i \leq n}\left\{\inf _{t \in \mathbb{T}} c_{i}(t)\right\}>0
$$

Then the linear system

$$
x^{\Delta}(t)=\operatorname{diag}\left(-c_{1}(t),-c_{2}(t), \ldots,-c_{n}(t)\right) x(t)
$$

admits an exponential dichotomy on $\mathbb{T}$.

By Lemma 3, we can get the following.

Lemma 5 Let $-C=\operatorname{diag}\left(-c_{1}(t),-c_{2}(t), \ldots,-c_{n}(t)\right)$, then $X(t)=e_{-C}\left(t, t_{0}\right)$ is a fundamental solution matrix of the linear system $x^{\Delta}(t)=\operatorname{diag}\left(-c_{1}(t),-c_{2}(t), \ldots,-c_{n}(t)\right) x(t)$.

\section{Existence of the unique almost periodic positive solution}

In this paper, we use the notation: for any bounded function $f(t)$, we denote $\bar{f}=\sup _{t \in \mathbb{T}} f(t)$, $\underline{f}=\inf _{t \in \mathbb{T}} f(t)$.

Throughout this paper, we assume that the bounded almost periodic functions $a(t), b_{i}(t)$, $\tau_{i}(t)$ satisfy $0<\underline{a} \leq a(t) \leq \bar{a}, 0<\underline{b_{i}} \leq b_{i}(t) \leq \overline{b_{i}}, 0<\underline{\tau_{i}} \leq \tau_{i}(t) \leq \overline{\tau_{i}},-a(t) \in \mathfrak{R}^{+}$and $N_{i}>0$ $(i=1,2, \ldots, m)$.

Due to biological significance, we restrict our attention to positive solutions of equation (1.2). The initial condition associated with equation (1.2) is given by

$$
x(t ; \phi)=\phi(t)>0 \quad \text { for } t \in\left[-\tau^{*}, 0\right]_{\mathbb{T}}, \tau^{*}=\max _{1 \leq i \leq m}\left\{\bar{\tau}_{i}\right\} .
$$

Let $X=\{w(t) \mid w \in C(\mathbb{T}, \mathbb{R}), w(t)$ is almost periodic function $\}$ with the norm $\|w\|=$ $\sup _{t \in \mathbb{T}}|w(t)|$, then $X$ is a Banach space. 
For $w \in X$, we consider the equation

$$
x^{\Delta}(t)=-a(t) x(t)+\sum_{i=1}^{m} \frac{b_{i}(t) w\left(t-\tau_{i}(t)\right)}{\left[1+w\left(t-\tau_{i}(t)\right)\right]^{N_{i}+1}} .
$$

Since $\inf _{t \in \mathbb{T}} a(t)=\underline{a}>0$, then from Lemma 4 we know that the linear equation $x^{\Delta}(t)=$ $-a(t) x(t)$ admits exponential dichotomy on $\mathbb{T}$.

Hence, by Lemma 2, we know that equation (3.1) has exactly one almost periodic solution

$$
x_{w}(t)=\int_{-\infty}^{t} e_{-a}(t, \sigma(s)) \sum_{i=1}^{m} \frac{b_{i}(s) w\left(s-\tau_{i}(s)\right)}{\left[1+w\left(s-\tau_{i}(s)\right)\right]^{N_{i}+1}} \Delta s .
$$

We define the operator $A: X \rightarrow X$,

$$
(A w)(t)=\int_{-\infty}^{t} e_{-a}(t, \sigma(s)) \sum_{i=1}^{m} \frac{b_{i}(s) w\left(s-\tau_{i}(s)\right)}{\left[1+w\left(s-\tau_{i}(s)\right)\right]^{N_{i}+1}} \Delta s, \quad w \in X .
$$

Obviously, $w(t)$ is the almost periodic solution of equation (1.2) if and only if $w$ is the fixed point of the operator $A$.

Let

$$
M_{i}=\frac{1}{N_{i}\left(1+\frac{1}{N_{i}}\right)^{N_{i}+1}} \quad(i=1,2, \ldots, m) .
$$

We make the following assumptions:

$\left(C_{1}\right)$ There exist two positive constants $\beta>\gamma>0$ such that

$$
\beta \geq \frac{1}{\underline{a}} \sum_{i=1}^{m} \overline{b_{i}} M_{i} \quad \text { and } \max _{1 \leq i \leq m}\left\{\frac{1}{N_{i}}\right\} \leq \gamma \leq \frac{1}{\bar{a}} \sum_{i=1}^{m} \frac{\beta \underline{b_{i}}}{(1+\beta)^{N_{i}+1}}
$$

$\left(\mathrm{C}_{2}\right)$

$$
\underline{a}>\sum_{i=1}^{m} \overline{b_{i}}
$$

Theorem 1 Assume that conditions $\left(\mathrm{C}_{1}\right)$ and $\left(\mathrm{C}_{2}\right)$ are satisfied, then equation (1.2) has a unique almost periodic positive solution.

Proof Let $\Omega=\{w \mid w \in X, \gamma \leq w(t) \leq \beta, t \in \mathbb{T}\}$.

Firstly, we prove that $A \Omega \subset \Omega$.

For $\forall w \in \Omega$, we have

$$
\begin{aligned}
(A w)(t) & =\int_{-\infty}^{t} e_{-a}(t, \sigma(s)) \sum_{i=1}^{m} \frac{b_{i}(s) w\left(s-\tau_{i}(s)\right)}{\left[1+w\left(s-\tau_{i}(s)\right)\right]^{N_{i}+1}} \Delta s \\
& \leq \int_{-\infty}^{t} e_{-a}(t, \sigma(s)) \sum_{i=1}^{m} \frac{\overline{b_{i}} w\left(s-\tau_{i}(s)\right)}{\left[1+w\left(s-\tau_{i}(s)\right)\right]^{N_{i}+1}} \Delta s
\end{aligned}
$$

For the function $f_{i}(x)=\frac{x}{(1+x)^{N_{i}+1}}, N_{i}>0, x \in[0,+\infty)$, it is easy to show $f_{i}^{\prime}(x)=\frac{1-N_{i} x}{(1+x)^{N_{i}+2}}$, which implies that $f_{i}(x)$ is increasing on $x \in\left[0, \frac{1}{N_{i}}\right]$ and decreasing on $x \in\left[\frac{1}{N_{i}},+\infty\right)(i=1,2, \ldots, m)$. 
So the function $f_{i}(x)=\frac{x}{(1+x)^{N_{i}+1}}, N_{i}>0, x \in[0,+\infty)$ reaches its maximum

$$
f_{i(\max )}=\frac{1}{N_{i}\left(1+\frac{1}{N_{i}}\right)^{N_{i}+1}}=M_{i} \quad \text { at } x=\frac{1}{N_{i}}(i=1,2, \ldots, m) .
$$

Hence we get

$$
\frac{w\left(s-\tau_{i}(s)\right)}{\left[1+w\left(s-\tau_{i}(s)\right)\right]^{N_{i}+1}} \leq M_{i}
$$

It follows from (3.2) that

$$
\begin{aligned}
(A w)(t) & \leq \int_{-\infty}^{t} e_{-a}(t, \sigma(s)) \sum_{i=1}^{m} \overline{b_{i}} M_{i} \Delta s=\sum_{i=1}^{m} \overline{b_{i}} M_{i} \int_{-\infty}^{t} e_{-a}(t, \sigma(s)) \Delta s \\
& \leq \sum_{i=1}^{m} \overline{b_{i}} M_{i} \int_{-\infty}^{t} e_{-\underline{a}}(t, \sigma(s)) \Delta s=\frac{1}{\underline{a}} \sum_{i=1}^{m} \overline{b_{i}} M_{i} \leq \beta
\end{aligned}
$$

On the other hand, we have

$$
\begin{aligned}
(A w)(t) & =\int_{-\infty}^{t} e_{-a}(t, \sigma(s)) \sum_{i=1}^{m} \frac{b_{i}(s) w\left(s-\tau_{i}(s)\right)}{\left[1+w\left(s-\tau_{i}(s)\right)\right]^{N_{i}+1}} \Delta s \\
& \geq \int_{-\infty}^{t} e_{-a}(t, \sigma(s)) \sum_{i=1}^{m} \frac{\underline{b_{i}} w\left(s-\tau_{i}(s)\right)}{\left[1+w\left(s-\tau_{i}(s)\right)\right]^{N_{i}+1}} \Delta s
\end{aligned}
$$

Note that

$$
\max _{1 \leq i \leq m}\left\{\frac{1}{N_{i}}\right\} \leq \gamma \leq w(t) \leq \beta \quad \text { for } t \in \mathbb{T}
$$

Since the function $f_{i}(x)=\frac{x}{(1+x)^{N_{i}+1}}\left(N_{i}>0\right)$ is increasing on $x \in\left[0, \frac{1}{N_{i}}\right]$ and decreasing on $x \in\left[\frac{1}{N_{i}},+\infty\right)$, then we have $f_{i}(w(t)) \geq f_{i}(\beta)$ for $t \in \mathbb{T}$.

That is,

$$
\frac{w(t)}{[1+w(t)]^{N_{i}+1}} \geq \frac{\beta}{(1+\beta)^{N_{i}+1}} \quad \text { for } t \in \mathbb{T} \text {. }
$$

So we get

$$
\frac{w\left(s-\tau_{i}(s)\right)}{\left[1+w\left(s-\tau_{i}(s)\right)\right]^{N_{i}+1}} \geq \frac{\beta}{(1+\beta)^{N_{i}+1}} .
$$

Thus by (3.4) we obtain

$$
\begin{aligned}
(A w)(t) & \geq \int_{-\infty}^{t} e_{-a}(t, \sigma(s)) \sum_{i=1}^{m} \frac{\beta \underline{b_{i}}}{(1+\beta)^{N_{i}+1}} \Delta s=\sum_{i=1}^{m} \frac{\beta \underline{b_{i}}}{(1+\beta)^{N_{i}+1}} \int_{-\infty}^{t} e_{-a}(t, \sigma(s)) \Delta s \\
& \geq \sum_{i=1}^{m} \frac{\beta \underline{b_{i}}}{(1+\beta)^{N_{i}+1}} \int_{-\infty}^{t} e_{-\bar{a}}(t, \sigma(s)) \Delta s=\frac{1}{\bar{a}} \sum_{i=1}^{m} \frac{\beta b_{i}}{(1+\beta)^{N_{i}+1}} \geq \gamma .
\end{aligned}
$$


Hence (3.3) and (3.5) imply

$$
\gamma \leq(A w)(t) \leq \beta
$$

In addition, $\forall w \in \Omega$, we know that equation (3.1) has exactly one almost periodic solution

$$
x_{w}(t)=\int_{-\infty}^{t} e_{-a}(t, \sigma(s)) \sum_{i=1}^{m} \frac{b_{i}(s) w\left(s-\tau_{i}(s)\right)}{\left[1+w\left(s-\tau_{i}(s)\right)\right]^{N_{i}+1}} \Delta s
$$

Since $x_{w}(t)$ is almost periodic, then $(A w)(t)$ is almost periodic.

This, together with (3.6), implies $A w \in \Omega$. So we have $A \Omega \subset \Omega$.

Now, we prove that $A$ is a contraction mapping on $\Omega$.

For $\forall u, w \in \Omega$, we have

$$
\begin{aligned}
\| A u & -A w \| \\
= & \sup _{t \in \mathbb{T}}|(A u)(t)-(A w)(t)| \\
= & \sup _{t \in \mathbb{T}}\left|\int_{-\infty}^{t} e_{-a}(t, \sigma(s))\left(\sum_{i=1}^{m} \frac{b_{i}(s) u\left(s-\tau_{i}(s)\right)}{\left[1+u\left(s-\tau_{i}(s)\right)\right]^{N_{i}+1}}-\sum_{i=1}^{m} \frac{b_{i}(s) w\left(s-\tau_{i}(s)\right)}{\left[1+w\left(s-\tau_{i}(s)\right)\right]^{N_{i}+1}}\right) \Delta s\right| \\
= & \sup _{t \in \mathbb{T}}\left|\int_{-\infty}^{t} e_{-a}(t, \sigma(s)) \sum_{i=1}^{m} b_{i}(s)\left(\frac{u\left(s-\tau_{i}(s)\right)}{\left[1+u\left(s-\tau_{i}(s)\right)\right]^{N_{i}+1}}-\frac{w\left(s-\tau_{i}(s)\right)}{\left[1+w\left(s-\tau_{i}(s)\right)\right]^{N_{i}+1}}\right) \Delta s\right| \\
& \leq \sup _{t \in \mathbb{T}} \int_{-\infty}^{t} e_{-a}(t, \sigma(s)) \sum_{i=1}^{m} \frac{u\left(s-\tau_{i}(s)\right)}{b_{i}}\left|\frac{w\left(s-\tau_{i}(s)\right)}{\left[1+u\left(s-\tau_{i}(s)\right)\right]^{N_{i}+1}}-\frac{\left.\left.\tau_{i}(s)\right)\right]^{N_{i}+1}}{[1+w(s-\tau}\right| \Delta s .
\end{aligned}
$$

By the mean value theorem, we have

$$
\begin{aligned}
& \left|\frac{u\left(s-\tau_{i}(s)\right)}{\left[1+u\left(s-\tau_{i}(s)\right)\right]^{N_{i}+1}}-\frac{w\left(s-\tau_{i}(s)\right)}{\left[1+w\left(s-\tau_{i}(s)\right)\right]^{N_{i}+1}}\right| \\
& =\left|f_{i}^{\prime}(\xi)\left[u\left(s-\tau_{i}(s)\right)-w\left(s-\tau_{i}(s)\right)\right]\right| \\
& =\left|\frac{1-N_{i} \xi}{(1+\xi)^{N_{i}+2}}\right|\left|u\left(s-\tau_{i}(s)\right)-w\left(s-\tau_{i}(s)\right)\right|,
\end{aligned}
$$

in which $\xi$ lies between $u\left(s-\tau_{i}(s)\right)$ and $w\left(s-\tau_{i}(s)\right)$.

Note that the function

$$
g_{i}(x)=\left|\frac{1-N_{i} x}{(1+x)^{N_{i}+2}}\right| \leq 1 \quad \text { for } x \in[0,+\infty)
$$

Thus we have

$$
\left|\frac{1-N_{i} \xi}{(1+\xi)^{N_{i}+2}}\right| \leq 1
$$

It follows from (3.8) that

$$
\left|\frac{u\left(s-\tau_{i}(s)\right)}{\left[1+u\left(s-\tau_{i}(s)\right)\right]^{N_{i}+1}}-\frac{w\left(s-\tau_{i}(s)\right)}{\left[1+w\left(s-\tau_{i}(s)\right)\right]^{N_{i}+1}}\right| \leq\left|u\left(s-\tau_{i}(s)\right)-w\left(s-\tau_{i}(s)\right)\right| .
$$


Hence, from (3.7) and (3.9), we get

$$
\begin{aligned}
\|A u-A w\| & \leq \sup _{t \in \mathbb{T}}\left\{\int_{-\infty}^{t} e_{-a}(t, \sigma(s)) \sum_{i=1}^{m} \overline{b_{i}}\left|u\left(s-\tau_{i}(s)\right)-w\left(s-\tau_{i}(s)\right)\right| \Delta s\right\} \\
& \leq \sup _{t \in \mathbb{T}}\left\{\int_{-\infty}^{t} e_{-a}(t, \sigma(s)) \sum_{i=1}^{m} \overline{b_{i}}\|u-w\| \Delta s\right\} \\
& \leq \sup _{t \in \mathbb{T}}\left\{\int_{-\infty}^{t} e_{-\underline{a}}(t, \sigma(s)) \sum_{i=1}^{m} \overline{b_{i}}\|u-w\| \Delta s\right\} \\
& =\sup _{t \in \mathbb{T}}\left\{\|u-w\| \sum_{i=1}^{m} \overline{b_{i}} \int_{-\infty}^{t} e_{-\underline{a}}(t, \sigma(s)) \Delta s\right\} \\
& =\sup _{t \in \mathbb{T}}\left\{\|u-w\| \frac{1}{a} \sum_{i=1}^{m} \frac{b_{i}}{\underline{a}}\right\}=\left(\frac{1}{a} \sum_{i=1}^{m} \overline{b_{i}}\right)\|u-w\| .
\end{aligned}
$$

Since $\frac{1}{a} \sum_{i=1}^{m} \overline{b_{i}}<1$, then we know that $A$ is a contraction mapping. Thus, by the contraction mapping fixed point theorem, the operator $A$ has a unique fixed point $w^{*}$ in $\Omega$. This means that equation (1.2) has a unique almost periodic positive solution $w^{*}(t)$, and $\gamma \leq w^{*}(t) \leq \beta$. The proof of Theorem 1 is completed.

\section{Global exponential stability of an almost periodic positive solution}

Theorem 2 Assume that conditions $\left(\mathrm{C}_{1}\right)$ and $\left(\mathrm{C}_{2}\right)$ are satisfied. Then equation (1.2) has a unique globally exponentially stable almost periodic positive solution.

Proof Since $\left(C_{1}\right)$ and $\left(C_{2}\right)$ are satisfied, then by Theorem 1 we know that equation (1.2) has a unique almost periodic positive solution $w^{*}(t)$, and $\gamma \leq w^{*}(t) \leq \beta$. Let $\psi(t)$ be the initial function of $w^{*}(t), w^{*}(t ; \psi)=\psi(t)$ for $t \in\left[-\tau^{*}, 0\right]_{\mathbb{T}}$. Now we prove that $w^{*}(t)$ is globally exponentially stable.

Suppose that $x(t)$ is an arbitrary positive solution of equation (1.2) with the initial function $x(t ; \phi)=\phi(t)>0, t \in\left[-\tau^{*}, 0\right]_{\mathbb{T}}$.

Let $y(t)=x(t)-w^{*}(t)$, then we have

$$
\begin{aligned}
y^{\Delta}(t)= & \left(x(t)-w^{*}(t)\right)^{\Delta} \\
= & -a(t) x(t)+\sum_{i=1}^{m} \frac{b_{i}(t) x\left(t-\tau_{i}(t)\right)}{\left[1+x\left(t-\tau_{i}(t)\right)\right]^{N_{i}+1}} \\
& -\left(-a(t) w^{*}(t)+\sum_{i=1}^{m} \frac{b_{i}(t) w^{*}\left(t-\tau_{i}(t)\right)}{\left[1+w^{*}\left(t-\tau_{i}(t)\right)\right]^{N_{i}+1}}\right) \\
= & -a(t)\left(x(t)-w^{*}(t)\right)+\sum_{i=1}^{m} \frac{b_{i}(t) x\left(t-\tau_{i}(t)\right)}{\left[1+x\left(t-\tau_{i}(t)\right)\right]^{N_{i}+1}} \\
& -\sum_{i=1}^{m} \frac{b_{i}(t) w^{*}\left(t-\tau_{i}(t)\right)}{\left[1+w^{*}\left(t-\tau_{i}(t)\right)\right]^{N_{i}+1}} .
\end{aligned}
$$


Let

$$
h(t)=\sum_{i=1}^{m} \frac{b_{i}(t) x\left(t-\tau_{i}(t)\right)}{\left[1+x\left(t-\tau_{i}(t)\right)\right]^{N_{i}+1}}-\sum_{i=1}^{m} \frac{b_{i}(t) w^{*}\left(t-\tau_{i}(t)\right)}{\left[1+w^{*}\left(t-\tau_{i}(t)\right)\right]^{N_{i}+1}},
$$

then it follows from (4.1) that

$$
y^{\Delta}(t)=-a(t) y(t)+h(t) .
$$

From (4.2), we know that $y(t)$ can be expressed as follows:

$$
y(t)=e_{-a}\left(t, t_{0}\right) y\left(t_{0}\right)+\int_{t_{0}}^{t} e_{-a}(t, s) h(s) \Delta s \quad\left(t \geq t_{0}\right), t_{0} \in\left[-\tau^{*}, 0\right]_{\mathbb{T}} .
$$

Thus, (4.3) implies that

$$
y(t)=e_{-a}\left(t, t_{0}\right)\left(\phi\left(t_{0}\right)-\psi\left(t_{0}\right)\right)+\int_{t_{0}}^{t} e_{-a}(t, s) h(s) \Delta s .
$$

Note that

$$
\begin{aligned}
|h(t)| & =\left|\sum_{i=1}^{m} b_{i}(t)\left(\frac{x\left(t-\tau_{i}(t)\right)}{\left[1+x\left(t-\tau_{i}(t)\right)\right]^{N_{i}+1}}-\frac{w^{*}\left(t-\tau_{i}(t)\right)}{\left[1+w^{*}\left(t-\tau_{i}(t)\right)\right]^{N_{i}+1}}\right)\right| \\
& \leq \sum_{i=1}^{m} b_{i}(t)\left|\frac{x\left(t-\tau_{i}(t)\right)}{\left[1+x\left(t-\tau_{i}(t)\right)\right]^{N_{i}+1}}-\frac{w^{*}\left(t-\tau_{i}(t)\right)}{\left[1+w^{*}\left(t-\tau_{i}(t)\right)\right]^{N_{i}+1}}\right| .
\end{aligned}
$$

By the mean value theorem, we have

$$
\begin{aligned}
& \left|\frac{x\left(t-\tau_{i}(t)\right)}{\left[1+x\left(t-\tau_{i}(t)\right)\right]^{N_{i}+1}}-\frac{w^{*}\left(t-\tau_{i}(t)\right)}{\left[1+w^{*}\left(t-\tau_{i}(t)\right)\right]^{N_{i}+1}}\right| \\
& \quad=\left|f_{i}^{\prime}(\eta)\left[x\left(t-\tau_{i}(t)\right)-w^{*}\left(t-\tau_{i}(t)\right)\right]\right| \\
& \quad=\left|\frac{1-N_{i} \eta}{(1+\eta)^{N_{i}+2}}\right|\left|x\left(t-\tau_{i}(t)\right)-w^{*}\left(t-\tau_{i}(t)\right)\right|,
\end{aligned}
$$

in which $\eta$ lies between $x\left(t-\tau_{i}(t)\right)$ and $w^{*}\left(t-\tau_{i}(t)\right)$.

Note that the function

$$
g_{i}(x)=\left|\frac{1-N_{i} x}{(1+x)^{N_{i}+2}}\right| \leq 1 \quad \forall x \in[0,+\infty)(i=1,2, \ldots, m) .
$$

Thus we have

$$
\left|\frac{1-N_{i} \eta}{(1+\eta)^{N_{i}+2}}\right| \leq 1
$$

From (4.6) we get

$$
\left|\frac{x\left(t-\tau_{i}(t)\right)}{\left[1+x\left(t-\tau_{i}(t)\right)\right]^{N_{i}+1}}-\frac{w^{*}\left(t-\tau_{i}(t)\right)}{\left[1+w^{*}\left(t-\tau_{i}(t)\right)\right]^{N_{i}+1}}\right| \leq\left|x\left(t-\tau_{i}(t)\right)-w^{*}\left(t-\tau_{i}(t)\right)\right| .
$$


Hence, by (4.5) and (4.7), we get

$$
|h(t)| \leq \sum_{i=1}^{m} b_{i}(t)\left|x\left(t-\tau_{i}(t)\right)-w^{*}\left(t-\tau_{i}(t)\right)\right| \leq\left\|x-w^{*}\right\| \sum_{i=1}^{m} \overline{b_{i}}
$$

It follows that

$$
\|h(t)\| \leq\left\|x-w^{*}\right\| \sum_{i=1}^{m} \overline{b_{i}}=\|y\| \sum_{i=1}^{m} \overline{b_{i}}
$$

Taking the norm on both sides of (4.4), we obtain

$$
\begin{aligned}
\|y(t)\| & \leq e_{-a}\left(t, t_{0}\right)\|\phi-\psi\|+\int_{t_{0}}^{t} e_{-a}(t, s)\|h(s)\| \Delta s \\
& \leq e_{-a}\left(t, t_{0}\right)\|\phi-\psi\|+\int_{t_{0}}^{t} e_{-a}(t, s)\|y\| \sum_{i=1}^{m} \overline{b_{i}} \Delta s .
\end{aligned}
$$

From (4.8), we get

$$
\frac{\|y(t)\|}{e_{-a}\left(t, t_{0}\right)} \leq\|\phi-\psi\|+\int_{t_{0}}^{t} \frac{\|y\|}{e_{-a}\left(s, t_{0}\right)} \sum_{i=1}^{m} \overline{b_{i}} \Delta s
$$

By the Gronwall inequality (see [8]), we obtain

$$
\frac{\|y(t)\|}{e_{-a}\left(t, t_{0}\right)} \leq\|\phi-\psi\| e_{\lambda}\left(t, t_{0}\right), \quad \text { here } \lambda=\sum_{i=1}^{m} \overline{b_{i}}
$$

Hence we get

$$
\begin{aligned}
\|y(t)\| & \leq\|\phi-\psi\| e_{\lambda}\left(t, t_{0}\right) e_{-a}\left(t, t_{0}\right) \\
& \leq\|\phi-\psi\| e_{\lambda}\left(t, t_{0}\right) e_{-\underline{a}}\left(t, t_{0}\right) \\
& =\|\phi-\psi\| e_{-(\underline{a}-\lambda)}\left(t, t_{0}\right) .
\end{aligned}
$$

That is,

$$
\left\|x(t)-w^{*}(t)\right\| \leq\|\phi-\psi\| e_{-(\underline{a}-\lambda)}\left(t, t_{0}\right), \quad \text { here } \underline{a}>\lambda,
$$

which means that $w^{*}(t)$ is globally exponentially stable. The proof of Theorem 2 is completed.

Remark If $\mathbb{T}=\mathbb{R}$ and $\mathbb{T}=\mathbb{Z}$, then equation (1.2) reduces to

$$
x^{\prime}(t)=-a(t) x(t)+\sum_{i=1}^{m} \frac{b_{i}(t) x\left(t-\tau_{i}(t)\right)}{\left[1+x\left(t-\tau_{i}(t)\right)\right]^{N_{i}+1}}, \quad t \in \mathbb{R}
$$


and

$$
x(k+1)-x(k)=-a(k) x(k)+\sum_{i=1}^{m} \frac{b_{i}(k) x\left(k-\tau_{i}(k)\right)}{\left[1+x\left(k-\tau_{i}(k)\right)\right]^{N_{i}+1}}, \quad k \in \mathbb{Z},
$$

respectively.

Our study unifies differential equations and difference equations. The explorations in this paper reveal that when we deal with the existence and stability of an almost periodic solution for differential equations and difference equations, it is unnecessary to prove results for differential equations and separately again for their discrete analogues (difference equations). We can unify such problems in the framework of dynamic equations on time scales.

\section{Competing interests}

The author declares that they have no competing interests.

\section{Author's contributions}

The author is entirely responsible for this research. The author read and approved the final manuscript.

\section{Acknowledgements}

The author expresses great thanks to the referees for their valuable comments and suggestions in improving the presentation of the manuscript. This work is supported by the Natural Science Foundation of Education Department of Anhui Province (KJ2014A043).

Received: 25 July 2014 Accepted: 20 January 2015 Published online: 11 February 2015

\section{References}

1. Kostitzin, VA: Symbiose, Parasitisme et Evolution. Hermann \& Cie, Paris (1943)

2. Hairston, NG: On the mathematical analysis of schistosome population. Bull. World Health Organ. 33, 45-62 (1965)

3. Tallis, GM, Leyton, MK: A schistosome approach to the study of parasite populations. J. Theor. Biol. 13, 160-172 (1966)

4. Leyton, MK: Stochastic models in population of hilmenthic parasites in the definitive host. II. Sexual mating functions. Math. Biosci. 39, 413-419 (1968)

5. May, R, Anderson, RM: Infectious Diseases of Humans: Dynamics and Control. Oxford University Press, Oxford (1995)

6. Elabbasy, EM, Saif, K, Saker, SH: Oscillation in host macroparasite model with delay time. Far East J. Appl. Math. 4 119-142 (2000)

7. Spedding, V: Taming nature's numbers. New Sci. 179(2404), 28-31 (2003)

8. Bohner, M, Peterson, A: Dynamic Equations on Time Scales: An Introduction with Applications. Birkhäuser, Boston (2001)

9. Bohner, M, Peterson, A: Advances in Dynamic Equations on Time Scales. Birkhäuser, Boston (2003)

10. Hilger, S: Analysis on measure chains - a unified approach to continuous and discrete calculus. Results Math. 18, 18-56 (1990)

11. Fink, A: Almost Periodic Differential Equations. Lecture Notes in Mathematics, vol. 377. Springer, Berlin (1974)

12. He, CY: Almost Periodic Differential Equations. Higher Education Press, Beijing (1992)

13. Li, YK, Wang, C: Almost periodic functions on time scales and applications. Discrete Dyn. Nat. Soc. 2011, 727068 (2011) doi:10.1155/2011/727068

14. Li, YK, Wang, C: Uniformly almost periodic functions and almost periodic solutions to dynamic equations on time scales. Abstr. Appl. Anal. 2011, 341520 (2011). doi:10.1155/2011/341520

15. Cheban, D, Mammana, C: Invariant manifolds, global attractors and almost periodic solutions of nonautonomous difference equations. Nonlinear Anal., Theory Methods Appl. 56(4), 465-484 (2004)

16. Zhang, J, Fan, M, Zhu, H: Existence and roughness of exponential dichotomies of linear dynamic equations on time scales. Comput. Math. Appl. 59(8), 2658-2675 (2010) 